\title{
Statut des ministres du culte et droit français
}

Jean-Marie Woehrling

\section{(2penEdition}

Journals

Édition électronique

URL : https://journals.openedition.org/rdr/431

DOI $: 10.4000 /$ rdr.431

ISSN : 2534-7462

\section{Éditeur}

Presses universitaires de Strasbourg

\section{Édition imprimée}

Date de publication : 14 novembre 2019

Pagination : 75-91

ISBN : 979-10-344-0054-6

ISSN : 2493-8637

\section{Référence électronique}

Jean-Marie Woehrling, "Statut des ministres du culte et droit français », Revue du droit des religions [En ligne], 8 | 2019, mis en ligne le 25 novembre 2019, consulté le 21 septembre 2021. URL : http:// journals.openedition.org/rdr/431 ; DOI : https://doi.org/10.4000/rdr.431

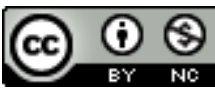

La revue du droit des religions est mise à disposition selon les termes de la Creative Commons Attribution - Pas d'Utilisation Commerciale 4.0 International - CC BY-NC 4.0. 


\section{STATUT DES MINISTRESDU CULTE ETDROIT FRANÇAIS}

\section{Jean-Marie WOEHRLING}

Ancien président de tribunal administratif

\section{Résumé}

La formule de «statut de ministre du culte» révèle en droit français un faible contenu normatif, attribuant une large marge de manœuvre aux institutions religieuses pour définir les règles applicables à leur personnel. Cette situation, perçue comme le corollaire du principe de neutralité de l'État, pourrait évoluer au regard des besoins qui se manifestent tant au plan d'une meilleure protection des agents des institutions religieuses que d'un renforcement de leurs garanties déontologiques. Des initiatives sont prises, mais ne font pas consensus. Diverses voies d'évolution restent à explorer.

\section{Abstract}

Behind the French concept of "statut de ministre du culte" (status of minister of religion), one finds only very few public regulations, which leaves a large discretion to religious organizations in defining the rules applicable to their staff. This situation respects the state neutrality towards religion but lets open questions concerning the protection of the personnel and the guaranties concerning their ethics. Initiatives are taken on these matters but satisfying solutions are still to be found. 
T a question du «statut des ministres des cultes» est fréquemment soulevée

Ldans l'actualité alors que pendant longtemps les discussions relatives au droit des cultes ne portaient guère sur le personnel religieux. La loi du 9 décembre 1905 concernant la séparation des Églises et de l'État se borne à poser le principe que "la République ne salarie aucun culte» (art. 2) et ne donne aucune précision sur le statut des agents des collectivités religieuses. La langue française comporte cependant la terminologie originale de «ministre du culte». Le questionnement relatif à cette notion s'est quelque peu renouvelé au regard du rôle grandissant joué par le culte musulman en France et les débats soulevés concernant la formation des imams ou les garanties de bonne intégration des responsables religieux dans la société française.

La présente étude vise à apporter quelques éléments de clarification à ce débat sur le plan juridique en établissant un rappel de l'état de droit actuel (1) et en examinant quelques hypothèses d'évolution (2). Dans ces développements, une attention particulière sera donnée à la situation des personnels religieux musulmans, puisque c'est d'eux dont il est fréquemment question dans la discussion contemporaine.

\section{L'ÉTAT DU DROIT}

Le recours aux termes de «statut des ministres du culte» est fréquent. Pourtant cette terminologie est trompeuse. Non seulement il n'existe pas de définition légale des «ministres du culte», mais encore le personnel religieux ne relève pas d'un cadre juridique légal élaboré que l'on pourrait qualifier valablement de statut. Cette situation laisse aux organisations cultuelles une grande latitude pour organiser ou ne pas organiser elles-mêmes, sous forme contractuelle ou par des règlements internes, le cadre juridique d'activité de leurs agents. 


\subsection{QU'EST-CE QU'UN MINISTRE DU CULTE (AU SENS DU DROIT FRANÇAIS) ${ }^{1}$ ?}

\section{Pas de définition légale}

Bien qu'il soit utilisé par différents textes ${ }^{2}$, le terme «ministre du culte» n'est défini ni par le droit écrit ${ }^{3}$, ni par la jurisprudence, ${ }^{4}$ ni même par la doctrine $e^{5}$.

Pourquoi cette absence de définition? On relève parfois qu'à défaut d'une définition légale du culte, il est également impossible de définir les ministres des cultes. On évoque aussi la nécessaire réserve de l'État et les exigences du principe de laïcité : il y a lieu de laisser à chaque culte le soin de s'organiser comme il l'entend en ce qui concerne son personnel. Le droit doit donc s'abstenir de préciser quels responsables de chaque culte peuvent se voir reconnaître comme «ministres du culte». Ces explications peuvent laisser insatisfait. La vraie raison semble plutôt résider dans le fait que la notion est un avatar historique et manque intrinsèquement de cohérence. L'explication la plus profonde de l'absence d'une définition claire et générale paraît être que la notion de «ministre du culte», qui est avant tout un héritage historique, est par elle-même incapable de correspondre à la diversité des situations actuelles.

1. Les développements qui suivent reprennent l'analyse que nous avons développée dans F. Messner, P.-H. Prélot, J.-M. Woehrling (dir.), Traité de droit français des religions, Paris, LexisNexis, $2^{\mathrm{e}}$ éd. 2013, n 2586 et $\mathrm{s}$.

2. En particulier par les textes maintenus en vigueur dans les départements d'Alsace et de Moselle, par certaines dispositions du Code civil (art. 909) et du Code pénal (art. 43321), ainsi que par la loi de 1905 (art. 30 à 35), par les textes particuliers relatifs à la couverture sociale des personnels religieux (CSS, art. L. 381-15 et s.). - Le rapporteur devant l'Assemblée nationale de la loi du 2 janvier 1978 relative aux régimes d'assurance maladie, maternité, invalidité, vieillesse applicable aux ministres des cultes et membres des congrégations et des collectivités religieuses avait relevé au sujet de l'utilisation de cette expression qu'il n'existait aucune définition du culte et donc du ministre du culte: JOAN, 19 déc. 1977, p. 8293.

3. Aucun texte de loi ni aucun règlement ne donnant une définition du ministre du culte, comme le relève A. Garay dans une étude sur «La situation légale du ministre du culte en France. Le cas des témoins de Jéhovah», RDP 1991, p. 1109.

4. Les décisions de justice appliquant les textes visant les ministres du culte n'ont pas eu besoin de définir ces derniers dans la mesure où cette qualité n'était pas discutée. Les instructions internes de la CAVIMAC, sans donner de définition du ministre du culte, essaient du moins de qualifier certaines situations telles que celles des séminaristes.

5. V. A. GARAY, art. cit. La doctrine administrative, à travers des circulaires de l'UNEDIC et des organes de la Sécurité sociale, a parfois essayé de préciser la notion de ministre du culte en recommandant l'assujettissement ou le non-assujettissement de certaines personnes à un régime social déterminé. 
Si, en pratique, on trouve en droit social des éléments de caractérisation du ministre du culte, ceux-ci sont largement inspirés de la conception du ministre telle qu'elle est envisagée dans le modèle catholique. En effet, les seules tentatives quelque peu élaborées de définition concernent les questions d'exclusion de cette catégorie de responsables religieux du droit du travail ou du régime de la sécurité sociale. Dans ce contexte, le ministre du culte se caractérise traditionnellement par un «sacerdoce» (mission religieuse particulière) dont l'intéressé est investi par une "consécration officielle » délivrée par une autorité religieuse supérieure par rapport à laquelle il est placé dans une situation d'obéissance hiérarchique, engagement qui est pris lors d'une cérémonie publique d'ordination.

Ces éléments de caractérisation peuvent difficilement servir de base pour la définition d'une catégorie «interreligieuse» de ministre du culte. Néanmoins, alors qu'ils n'ont aucun fondement juridique précis, ils ont été retenus par la jurisprudence ${ }^{6}$ et par la doctrine administrative ${ }^{7}$ par une sorte de généralisation des caractères distinctifs du clerc catholique.

\section{Inadéquation de la notion}

Il existe d'autres formes d'acteurs religieux que celle qui se rapproche du statut traditionnel des «clercs», au sens de personnes spécialisées dans des fonctions sacerdotales. Non seulement la position des permanents religieux des autres cultes ne se recoupe guère avec le modèle du prêtre catholique, mais même au sein de l'Église catholique se développent des statuts originaux, tels les "animateurs pastoraux», qui ne peuvent trouver que difficilement leur place dans les cadres conceptuels classiques. L'état du droit se montre donc, pour une large part, incapable de donner un cadre cohérent et clair à la diversité des situations concrètes en présence.

Cela paraît vrai notamment pour le personnel religieux musulman. La vision française tend à imaginer des sortes de prêtres musulmans, que seraient les imams, alors que ces derniers correspondent à des traditions et à des fonctions tout à fait différentes. En, fait, plutôt que sur le statut des «ministres

6. Ainsi, la cour de Douai, dans un arrêt du 30 mai 1984, formule que «la préparation du règne de Dieu sur terre, ne constitue pas du fait de sa finalité spirituelle, une activité relevant du Code du travail» (CA Douai, 5e ch. soc., 30 mai 1984: JCP G 1986, II, 20628 note Th. REVET, arrêt confirmé par Cass. soc., 20 nov. 1986, n² 2804).

7. Circulaires de l'UNEDIC ou de la CAVIMAC. Selon les services de gestion de la CAVIMAC, est considéré comme ministre du culte le séminariste ou le novice qui a prononcé solennellement lors d'une cérémonie publique un premier engagement consigné par écrit (lettre min. 23 mars 1988). 
du culte», il faudrait s'interroger sur les règles juridiques applicables aux «agents des organisations religieuses».

\subsection{QU'EST-CE QU'UN STATUT (LÉGAL) ?}

S'il n'y a pas de définition claire des ministres du culte, il n'y a pas non plus de conception précise du «statut» auquel ces personnes seraient soumises en droit français.

Un statut désigne en général un ensemble de règles cohérentes organisant l'ensemble de la situation juridique d'une catégorie définie de personnes: formation; recrutement; conditions d'exercice de l'activité; droits et obligations (incompatibilités, etc.); mécanismes de contrôle et protection; situation matérielle et financière; position par rapport au droit du travail et au droit de la protection sociale; sanctions; révocation. Or, même en admettant que la catégorie des ministres des cultes puisse être approximativement circonscrite, il n'y a pas de corps substantiel de règles qui les concerne et définissent un statut légal (sauf peut-être pour les ministres des cultes statutaires propres à l'Alsace-Moselle).

\subsection{QUELLES RÈGLES LÉGALES VISENT SPÉCIFIQUEMENT LES « MINISTRES DU CULTE »?}

On ne peut concrètement repérer que quatre catégories de règles législatives très parcellaires concernant les «ministres des cultes » en droit français général:

- l'article 909 du Code civil: les ministres du culte ne peuvent profiter de dons ou de legs de la part des personnes auxquelles ils ont apporté assistance durant la maladie qui a conduit à leur décès

- l'article 433-21 du Code pénal: le ministre d'un culte qui procédera, de manière habituelle, aux cérémonies religieuses de mariage sans que ne lui ait été justifié l'acte de mariage préalablement reçu par les officiers de l'état civil sera puni pénalement

- les articles 34 et 35 de la loi du 9 décembre 1905 prévoyant des sanctions pénales pour tout ministre d'un culte qui, dans les lieux où s'exerce ce culte, aura publiquement outragé ou diffamé un citoyen chargé d'un service public ou prononcé un discours ou affiché voire distribué un écrit dans les lieux où s'exerce le culte, contenant une provocation directe à résister à l'exécution des lois ou aux actes légaux de l'autorité publique, ou à soulever ou à armer une partie des citoyens contre les autres. 
- la loi du 2 janvier 1978 relative aux régimes d'assurance maladie, maternité, invalidité, vieillesse applicable aux ministres des cultes et membres des congrégations et des collectivités religieuses, codifiée aux articles L. 382-15 et s. du Code de la sécurité sociale. Cette législation prévoit une couverture maladie et retraite pour les ministres des cultes qui ne disposent pas de contrat de travail. C'est une protection acceptable en matière de maladie, mais très limitée sur le plan de la retraite. Il y est recouru par des personnels religieux du culte musulman de manière marginale seulement (3\% du total des personnes concernées). La doctrine administrative et la jurisprudence relatives à la mise en œuvre de cette loi ont tenté de préciser la notion de culte ou le point de départ d'appartenance à une communauté religieuse, mais elles n'apportent guère de précision sur le statut des ministres du culte.

Enfin, il existe non pas une loi, mais des jurisprudences qui visent certains ministres du culte: une jurisprudence exclut pour certains d'entre eux l'application du droit du travail, alors même que les critères usuels du contrat de travail sont réunis ${ }^{8}$. Cette conception de la mission spirituelle spécifique du ministre du culte a été étendue aux imams musulmans dès lors que leur mission exclusive ou principale est spirituelle ${ }^{9}$. Cette solution n'est cependant pas d'application stable: si les conditions de l'existence d'un contrat de travail sont réunies, les juridictions vont fréquemment appliquer celui-ci nonobstant le fait que le cocontractant exerce une mission spirituelle ${ }^{10}$. Si les conditions

8. S'agissant des prêtres catholiques et des pasteurs relevant de la Fédération protestante de France, la Cour de cassation estime qu'il n'y a pas lieu de rechercher si les critères du contrat de travail sont réunis (Cass. soc., 28 avr. 2011, n 09-72.721, Mme X: «les pasteurs des églises et œeuvres cultuelles relevant de la Fédération protestante de France ne concluent pas, relativement à l'exercice de leur ministère, un contrat de travail avec les associations cultuelles légalement établies»; par conséquent en se fondant sur le fait que Mme X, qui «exerçait un ministère de pasteur assurant le culte au sein d'une paroisse pour une association cultuelle de l'Église évangélique luthérienne de France» avait été embauchée par « contrat écrit régularisé d'une déclaration unique d'embauche», la cour d'appel a, par fausse application, violé les textes applicables, «peu important [sic] que les rapports entre les parties aient été formalisés par une déclaration d'embauche et un contrat de travail, susvisés».)

9. Cass. soc., 6 mars 1986, Farfour: JCP E 1986, act. 15414; 6 mai 2009, Achour c. Ass. cultuelle et culturelle de la communauté musulmane de l'agglomération d'Annecy: JurisData $\mathrm{n}^{\circ}$ 2009-048108; CA Paris, 18 sept. 2007, $\mathrm{n}^{\circ}$ S06/00664, Selali c. Ass. culturelle et cultuelle de Sens et sa région: JurisData ${ }^{\circ}$ 2007-342314; CA Caen, 28 oct. 2005, $\mathrm{n}^{\circ}$ 04/01155, At Lachaikh c. Ass. islamique et culturelle du Calvados: JurisData $\mathrm{n}^{\circ}$ 2005290139; CA Paris, 3 juin 2004, n S04/30802, Posavljak c. Centre culturel bosniaque: JurisData $\mathrm{n}^{\circ}$ 2004-246075.

10. Les conditions d'emploi des imams peuvent être ambivalentes: une personne peut être appelée à exercer les fonctions exclusives d'imam d'une communauté musulmane tout en étant engagée fictivement comme animateur culturel par l'association constituée par cette communauté (V. CA Colmar, 8 mars 2016, n 14/01850, concernant l'emploi d'un imam par l'association socioculturelle Espérance gérant la mosquée de Saint-Louis). 
d'existence du contrat de travail ne sont pas établies, l'on reconnaîtra l'existence d'une activité spécifique pour les imams musulmans, dès lors que leur mission exclusive ou principale est spirituelle. Concrètement, il n'est guère compréhensible que la jurisprudence applique les critères usuels du contrat de travail pour certains responsables religieux et les rejette pour d'autres.

Une autre jurisprudence admet que, même si l'existence d'un contrat de travail est reconnue pour un ministre du culte, celui-ci peut être soumis à des règles spécifiques de loyauté par rapport à l'institution qui l'emploie; toutefois, cette dernière jurisprudence n'est pas propre aux ministres des cultes: elle concerne toutes les personnes engagées par des entreprises de «tendance» dès lors que la mission qui leur est confiée suppose une obligation de loyauté particulièrement rigoureuse ${ }^{11}$.

Ces quelques règles ne constituent pas un «statut», mais seulement des dispositions éparses relatives aux ministres des cultes.

\subsection{COMMENT ANALYSER LA SITUATION DES MINISTRES DU CULTE DANS UN CONTEXTE DE LARGE ABSENCE DE RÈGLES LÉGALES SPÉCIFIQUES?}

Comme il n'y a formellement pas de «lacunes» en droit, on peut considérer qu'il existe bien un statut des ministres des cultes, mais un statut caractérisé par une parfaite liberté: pas de conditions de formation, de recrutement ou de nomination, pas de conditions propres de déroulement de carrière ou de révocation, pas de protections ou d'obligations particulières en dehors des quelques règles mentionnées précédemment.

Cette liberté fait que c'est aux autorités religieuses de définir elles-mêmes le «statut», c'est-à-dire le régime juridique applicable à leurs «ministres du culte» sur la base de leur pouvoir d'organisation et d'accords contractuels. Les autorités religieuses peuvent combler en effet elles-mêmes une partie de ce «vide» juridique et développer des règles, qui leur sont propres, de formation et d'emploi de leur personnel. Elles sont donc compétentes pour décider des conditions de nomination ou de révocation de ministres du culte ou pour imposer une sanction ecclésiastique. Traditionnellement, les autorités publiques n'interviennent pas par rapport à ces règles d'organisation interne des cultes et les tribunaux refusent de connaître des litiges qui peuvent en naître.

11. Sur ce concept d'entreprise de tendance, V. F. Messner, P.-H. Prélot, J.-M. Woehrling (dir.), op.cit., $\mathrm{n}^{\circ} 1881$ et $\mathrm{s}$. 
L'efficacité d'une telle réglementation interne dépend beaucoup de l'existence d'une hiérarchie reconnue et d'une discipline observée à l'intérieur des mouvements religieux. Certes, une simple organisation locale peut elle aussi fixer des règles internes quant au statut de ses ministres du culte. Mais ce procédé n'a d'effet véritablement structurant que si une organisation est reconnue comme exerçant un rôle d'harmonisation pour l'ensemble d'un culte. C'est le cas pour les grandes organisations religieuses traditionnelles (Église catholique, Fédération protestante ${ }^{12}$ ).

C'est ce qui a été tenté avec les travaux relatifs à une charte des imams ${ }^{13}$ incluant une convention-type d'emploi de ministre du culte musulman. Cependant avec un succès relatif car les documents élaborés ont été mis en cause par une partie des organisations musulmanes ${ }^{14}$. La charte porte essentiellement sur des principes déontologiques. Le modèle de convention destiné à lier une association gestionnaire du culte musulman avec un «ministre du culte» exerçant les fonctions d'imam définit les missions de ce dernier et précise qu'il est placé «sous la responsabilité de l'association» qui lui verse une rémunération, sans qu'il ait la qualité de salarié, sa protection sociale relevant de la CAVIMAC. L'application de ces documents relève du choix de chaque association locale et il faut attendre pour connaître la manière dont ils seront mis en œuvre par les tribunaux.

\section{La dépendance par rapport à des statuts d'emploi de droit étranger}

Il faut aussi relever qu'un nombre important de responsables religieux musulmans sont au service d'autorités étrangères (Maroc, Turquie...) qui définissent leurs conditions d'intervention et assurent leur rémunération. Ces

12. Le Code de droit canonique, la constitution de l'Église protestante unie de France, le règlement du Consistoire israélite de 1972.

13. Document adopté le 29 mars 2017 par le Conseil français du culte musulman (CFCM): https://www.ethik-life.com/wp-content/uploads/2017/05/343503789-Le-CFCM-adopteune-charte-de-l-imam.pdf [consulté le 15 mai 2019].

14. Pour l'Union des mosquées de France (UMF) : «la mise en place d'une procédure d'agrément ou de recommandation, permettant d'aider les mosquées dans le recrutement de leurs imams et de s'assurer de la qualité de leur formation théologique et profane, doit être au centre des préoccupations du culte musulman", une procédure qui serait initiée par une commission rattachée au Conseil départemental des imams et qui repose sur "l'adhésion des imams à une charte de valeurs" - plus élaborée que celle contestée du CFCM - "et l'élaboration d'une convention-type, la plus explicite possible, définissant les contours de la mission de l'imam et de son statut et de préciser davantage ses liens avec l'association gestionnaire de la mosquée" »: H. Ben RhoumA, «Organisation et financement du culte musulman: l'UMF plaide pour la départementalisation », SaphirNews, 31 mai 2018: https:// www.saphirnews.com/Organisation-et-financement-du-culte-musulman-l-UMF-plaidepour-la-departementalisation_a25248.html [consulté le 15 mai 2019]. 
personnes ont donc un véritable statut, mais fixé par des autorités étrangères. En l'état actuel du droit, cette pratique n'est pas irrégulière, sous réserve pour les intéressés d'être titulaires d'un titre de séjour.

Pour conclure, on peut constater que les conditions de recrutement et le régime juridique des agents des organisations religieuses sont faiblement déterminés par le droit, le concept de ministre du culte étant lui-même en grande partie indéfini. Cela laisse une large place d'auto-organisation aux institutions religieuses. Dans le cas du culte musulman, cette faculté d'autoorganisation est encore peu utilisée, sauf en ce qui concerne le personnel dépendant d'instances étrangères.

\section{2. ÉLÉMENTS D’APPRÉCIATION ET PERSPECTIVES D'ÉVOLUTION}

La situation que l'on vient de décrire est-elle satisfaisante et l'État pourrait-il ou devrait-il intervenir pour régler de manière plus précise les règles statutaires applicables aux ministres des cultes ou, de façon plus générale, aux agents des institutions religieuses? La question est soulevée notamment au sujet des personnels du culte musulman, pour lesquels on évoque régulièrement certaines insuffisances de formation (concernant les institutions et la culture françaises, mais parfois aussi concernant les préceptes du culte musulman eux-mêmes) ou des garanties incertaines au regard du risque de positions intégristes ou radicales. De manière plus générale, dans une société marquée par un souci de régulation et de garanties dans tous les domaines, ne paraît-il pas légitime que ces préoccupations de protection s'appliquent également dans le domaine des institutions religieuses? Sur un autre plan, qui intéresse toutes les organisations religieuses, on s'inquiète de plus en plus des limitations apportées aux garanties des personnels des institutions religieuses au regard du pouvoir de leurs employeurs. Là encore se pose la question d'une réglementation plus précise.

Sans prendre parti sur la pertinence de ces préoccupations, on se bornera dans la présente étude essentiellement juridique à examiner la question des conditions et limites légales d'une intervention plus prononcée de la puissance publique en ce qui concerne les règles applicables aux agents des collectivités religieuses. Il nous faut d'abord préciser les principes que doit respecter un régime juridique destiné à mieux définir le «statut des ministres des cultes» ou de façon plus générale le cadre juridique des agents des collectivités religieuses. Puis on évoquera quelques perspectives d'évolution. 


\subsection{LES PRINCIPES À RESPECTER}

À cet égard, la question des règles applicables aux agents des institutions religieuses est balisée par trois principes juridiques.

\subsubsection{LE PRINCIPE DE LA LIBERTÉ RELIGIEUSE}

Ce principe consacré par la Convention européenne des droits de l'homme et par la Constitution vient limiter l'intervention de l'État dans l'organisation des cultes en ce qui concerne la gestion de leurs personnels religieux. Il implique le droit pour toutes les organisations religieuses de s'organiser conformément à leur «autocompréhension». Ce principe de l'autonomie des organisations religieuses s'applique notamment aux conditions d'activité de leurs personnels. Un cadre juridique qui imposerait que les personnels religieux soient placés dans des conditions d'emploi incompatibles avec les conceptions de l'engagement religieux de tel ou tel culte serait contraire au principe de l'autonomie d'organisation des cultes et par suite, à la liberté de religion. La Cour européenne des droits de l'homme a réaffirmé à de nombreuses occasions le principe de l'autonomie d'organisation des institutions religieuses, par exemple en ce qui concerne leur droit de déterminer les conditions d'engagement ou de licenciement de leur personnel religieux ${ }^{15}$, ou en ce qui concerne la possibilité d'exclure le droit syndical pour ce personnel ${ }^{16}$. Si le principe constitutionnel de la liberté du culte tel que le Conseil constitutionnel le déduit de l'article 10 de la Déclaration des droits de l'homme de $1789^{17}$ n'a pas encore donné lieu à une application dans le domaine du droit applicable au personnel religieux en France, on peut cependant considérer qu'il inclut le principe de l'autonomie des organisations religieuses. On trouve d'ailleurs une mention de ce principe dans l'article 4 de la loi du 9 décembre 1905 sur la séparation de l'État et des Églises ${ }^{18}$. Le législateur a montré par exemple son souci de ne pas imposer un régime de sécurité sociale à un culte qui y était opposé ${ }^{19}$. Par ailleurs, sans base légale particulière, la jurisprudence

15. CEDH, Gde ch., 12 juin 2014, n 56030/07, Fernández Martínez c. Espagne.

16. CEDH, Gde ch., 9 juill. 2013, n 2330/09, Sindicatul "Păstorul cel Bun» c. Roumanie.

17. Cons. const., 7 oct. 2010, $\mathrm{n}^{\circ}$ 2010-613 DC, Loi interdisant la dissimulation du visage dans l'espace public.

18. Cet article limite le transfert des biens d'églises «aux associations qui, en se conformant aux règles d'organisation générale du culte dont elles se proposent d'assurer l'exercice, se seront légalement formées ». Il pose la règle de la reconnaissance par l'État des règles d'organisation générale de chaque culte.

19. La loi «Viatte» du 19 février 1950 dispose que «l'exercice du ministère du culte catholique n'est pas considéré comme une activité professionnelle au regard de la législation sociale, en tant qu'il se limite à une activité exclusivement religieuse». 
de la Cour de cassation est dominée par l'idée du respect des conceptions propres au culte catholique quant au statut du prêtre.

Ce respect du principe de l'autonomie des organisations religieuses en ce qui concerne la gestion de leurs personnels n'est cependant pas absolu. Le droit de l'État doit offrir un cadre suffisamment adapté, mais n'est pas tenu de renoncer à ses propres impératifs. La liberté de religion peut entrer en conflit avec d'autres principes, notamment en ce qui concerne la protection des personnes employées dans des fonctions religieuses et le respect de l'ordre public. C'est dans la détermination de ces limites et de la nécessaire conciliation entre des principes concurrents que se situent les véritables difficultés.

Une façon de résoudre ces difficultés peut consister dans une méthode visant pour l'État non pas à imposer unilatéralement aux cultes, ou à un culte, des conditions d'organisation, notamment quant à l'emploi de personnel religieux, mais à obtenir l'engagement d'un culte à appliquer à son personnel certaines règles (règles de formation, règles de comportement, etc.) en échange de certaines facilités consenties (notamment au plan financier). Mais ces arrangements doivent respecter le principe de neutralité.

\subsubsection{LE PRINCIPE DE LA NEUTRALITÉ DE L'ÉTAT}

Le principe de la neutralité de l'État, et sa version française à savoir le principe de laïcité, ont parfois été compris comme exigeant d'appliquer «le droit commun» aux institutions religieuses. Cette conception n'a pas été étendue au droit des personnels religieux dont la situation particulière ne peut être ignorée. La neutralité bien comprise implique même une retenue dans l'ingérence éventuelle de l'État dans les règles d'emploi de personnel religieux.

La Cour européenne des droits de l'homme considère d'ailleurs que le principe de neutralité religieuse de l'État justifie que celui-ci ne s'implique pas dans des critères d'emploi du personnel religieux, tels que le célibat ${ }^{20}$. En principe, les institutions religieuses sont libres d'engager et de révoquer leur personnel comme elles l'entendent sans contrôle des pouvoirs publics et donc sans être par exemple soumises aux règles de droit commun de nondiscrimination ${ }^{21}$. Lorsque les autorités publiques sont impliquées à un titre ou à un autre (pouvoir de nomination ou d'agrément en droit alsacien-mosellan ou pour des aumôniers de prisons ou d'hôpitaux, etc.), elles doivent limiter

20. CEDH, Gde ch., 12 juin 2014, n 56030/07, Fernández Martínez c. Espagne.

21. Dir. $n^{\circ}$ 2000/78/CE, 27 nov. 2000, portant création d'un cadre général en faveur de l'égalité de traitement en matière d'emploi et de travail; C. trav., art. L. 1133-1. 
leur intervention de manière à ne pas empiéter sur le fonctionnement interne des organisations religieuses ${ }^{22}$.

Le principe de laïcité, tel qu'il est interprété par le Conseil constitutionnel $^{23}$, exclut que l'État «salarie» un culte, donc rémunère du personnel religieux. Il faut comprendre cette restriction comme interdisant à l'État de rémunérer du personnel pour des missions religieuses définies par les organisations concernées. Mais l'État peut engager et donc rémunérer des personnes investies de responsabilités religieuses pour des missions qu'il leur attribue lui-même, que ces misions aient un objet non religieux (enseignement, etc.) ou même religieux (aumôniers d'hôpitaux ou de prisons). Dans ces derniers cas, pour concilier l'autonomie des institutions religieuses concernées et la neutralité de l'État, le responsable religieux est placé sous une sorte de double tutelle concrétisée par une lettre de mission de l'institution religieuse et par un acte d'engagement de l'autorité publique.

Mais le principe de la neutralité de l'État n'exclut pas que les pouvoirs publics fixent des conditions à l'exercice de certaines activités religieuses, par exemple des conditions de diplôme, si ces conditions sont justifiées par un motif d'intérêt général, sans pour autant représenter un obstacle au fonctionnement de l'organisation religieuse. Pour le Conseil constitutionnel, le principe de laïcité signifie qu'aucun groupe ne peut «se prévaloir de ses croyances religieuses pour s'affranchir des règles communes régissant les relations entre collectivités publiques et particuliers ${ }^{24} »$. On peut comprendre cette jurisprudence comme signifiant que si l'intérêt général exige certaines formes d'organisation et de contrôle pour les responsables religieux qui exercent une influence sur les consciences ou la psychologie des personnes, ces règles peuvent également s'appliquer aux groupements religieux, dès lors que les mesures prises sont nécessaires et proportionnées. Les pouvoirs publics ne peuvent rester insensibles au choix des personnels investis de l'influence considérable qui peut s'attacher à l'exercice de responsabilités religieuses. Ceux-ci doivent présenter des garanties, comme c'est le cas pour

22. CE, 17 oct. 1980, Pont: Lebon, p. 374; AJDA, 1981, p. 256, concl. Labetoulle. - CE, 27 mai 1994, Bourges: Lebon, p. 263. - Pour une illustration plus récente, V. CAA Nancy, 26 sept. 2006, $\mathrm{n}^{\circ}$ 04NC01127, Bruno c. Centre hospitalier de Mulhouse: «la décision par laquelle l'archevêque de Strasbourg a décidé de mettre fin à la mission qu'il avait confiée à Mlle X d'exercer les fonctions d'aumônier au Centre hospitalier de Mulhouse ne constitue pas une mesure susceptible d'être discutée devant la juridiction administrative».

23. Cons. const., 21 févr. 2013, n²012-297 QPC, Association pour la promotion et l'expansion de la laïcité.

24. Cons. const., 19 nov. 2004, n²004-505 DC, Traité établissant une Constitution pour l'Europe. 
d'autres responsables sociaux ou professionnels. De même, l'influence et l'autorité exercées par des responsables religieux justifient le droit de les soumettre à des règles particulières, et notamment à des exigences spéciales en matière de moralité, d'obligation de secret, etc. ${ }^{25}$.

C'est dans cet esprit que l'obligation d'obtention d'un diplôme de formation civile et civique a été créée en 2017 en vue d'assurer la formation des aumôniers militaires, hospitaliers et pénitentiaires. Il est délivré dans le cadre des universités publiques ou privées (instituts catholiques) ${ }^{26}$. Ce texte a été considéré par le Conseil d'État comme conforme à la liberté religieuse et à l'article 10 de la Déclaration de droits de l'homme et du citoyen ${ }^{27}$. Le juge administratif a relevé qu'en imposant une obligation de détention d'un diplôme de formation civile et civique pour les aumôniers, le pouvoir réglementaire a créé une condition au recrutement de ces aumôniers qui repose sur la poursuite d'objectifs d'intérêt général et de sauvegarde de l'ordre public en lien avec la mission de ceux-ci. L'institution d'une telle condition n'a pas pour effet d'encadrer l'exercice des cultes ni de substituer l'appréciation de l'Administration à celle des autorités cultuelles, auxquelles il appartient de proposer les candidats aux fonctions d'aumônier. La formation en matière civile et civique visée par le décret ne porte pas sur leur ministère religieux, mais sur l'environnement social, institutionnel et juridique dans lequel s'exerce leur activité d'aumônier et n'implique pas que l'Administration porte une appréciation sur le contenu des croyances concernées. Dans ces conditions, le moyen tiré de ce qu'en instituant cette condition de diplôme le pouvoir réglementaire se serait immiscé dans l'organisation des cultes a été écarté.

L'État peut aussi faire usage de la législation relative à l'entrée des étrangers sur le sol national à l'encontre d'agents religieux que des organisations religieuses souhaiteraient employer. Sur la base de cette législation de police, l'activité de ressortissants étrangers comme personnels religieux en France peut être soumise à des conditions spécifiques qui peuvent comporter notamment des règles relatives à la formation ou aux diplômes de ces personnels ${ }^{28}$.

25. Il y a peu de jurisprudence de la Cour européenne des droits de l'homme sur ce point. Ses décisions ont fréquemment porté sur la légitimité d'une non-intervention de l'État. Mais on peut penser que, comme pour la question du port du foulard, elle laisse aux États une marge d'appréciation quant aux nécessités impliquées par un contexte national.

26. D. $n^{\circ}$ 2017-756, 3 mai 2017, relatif aux aumôniers militaires, hospitaliers et pénitentiaires et à leur formation civile et civique.

27. CE, 27 juin 2018, n 412039: V. P.-H. PrÉLOT, « La laïcité devant le Conseil d'État en $2018 »$, Revue du droit des religions, n 7, 2019, p. 209-220.

28. S. JouAnneau, Les imams en France, une autorité religieuse sous contrôle, Marseille, Agone, 2013; «Régulariser ou non un imam étranger en France: droit au séjour et définition 
Si des conditions d'accès et des contrôles peuvent être regardés comme nécessaires pour certaines fonctions religieuses, l'État peut également souhaiter, en application du principe de neutralité, laisser aux autorités religieuses elles-mêmes le soin d'exercer la définition et le contrôle du respect de ces garanties, en estimant que ces autorités justifient d'une représentativité et d'une responsabilité suffisante.

\subsubsection{LA PROTECTION DES PERSONNELS RELIGIEUX}

Du fait de l'autonomie conférée aux organisations religieuses, les personnels de ces organisations ne bénéficient pas des mêmes avantages et protections que les agents d'organisations civiles ou économiques traditionnelles. Ces agents sont censés adhérer aux convictions des organisations religieuses dont ils ont accepté les règles. Si leur comportement ou l'expression de leurs opinions n'est plus en conformité avec les attentes de l'organisation religieuse, celle-ci peut les démettre. Comme indiqué précédemment, l'organisation religieuse peut aussi développer des critères de sélection pour l'engagement ou la promotion qui peuvent déroger à des règles de non-discrimination (sexe, préférences sexuelles, état matrimonial, etc.) si ces critères sont en relation avec des convictions religieuses ${ }^{29}$. Une organisation religieuse peut aussi choisir un autre cadre d'emploi que le salariat, même si l'activité de l'agent religieux est permanente et que celui-ci exerce une responsabilité de type professionnelle sous la responsabilité d'un supérieur. La protection sociale de l'agent religieux peut de ce fait être précaire.

Ces situations ${ }^{30}$, qui sont pour l'essentiel celles de toutes les entreprises de "tendance», étaient autrefois acceptées sans grande contestation aussi bien par les intéressés eux-mêmes que par les commentateurs ou par le législateur. Aujourd'hui, on considère cependant que certaines garanties et protections minimales doivent être fournies aux personnels religieux. Ce courant reste encore timide. La Cour européenne des droits de l'homme évolue dans ce sens, même si elle tend encore à privilégier l'autonomie

du "bon imam" en pays laique», Politix, n 2, 2009, p. 147-166: https://www-cairn-info. scd-rproxy.u-strasbg.fr/revue-politix-2009-2-page-147.htm [consulté le 15 mai 2019].

29. L'article 4 al. 2 de la directive 2000/78/CE du 27 novembre 2000 portant création d'un cadre général en faveur de l'égalité de traitement en matière d'emploi et de travail précise que les États membres peuvent autoriser les organisations religieuses à prendre en compte dans le cadre de leurs activités professionnelles les convictions de leurs agents lorsqu'elles constituent une exigence professionnelle essentielle et légitime.

30. Sans qu'il paraisse utile de distinguer entre les agents des organisations religieuses en général ou les «ministres du culte» en particulier. 
d'organisation des entreprises religieuses ${ }^{31}$. En particulier, elle exige que les instances de recours examinent avec sérieux l'ensemble des éléments du litige et notamment vérifient si le motif de licenciement est en rapport suffisant avec la fonction exercée. La jurisprudence française manifeste une tendance similaire $^{32}$, même si elle reste sensible aux prérogatives des autorités religieuses. Une protection accrue du personnel religieux au regard de mesures disciplinaires détachables des responsabilités religieuses exercées pourrait donc être considérée comme compatible avec le principe de l'autonomie des organisations religieuses.

Liberté religieuse, neutralité et droit d'intervention de l'État, protection des agents religieux, sont des éléments qui entrent souvent en conflit et qu'il faut essayer de concilier. Le droit français ne propose pas toujours des solutions qui prennent en compte de manière satisfaisante ces impératifs contradictoires dans le sens d'une conciliation équilibrée. Au total, ces trois principes définissent un champ d'action fortement contraint, mais non dépourvu de marge d'action. La tendance encore incertaine va dans le sens d'une plus grande responsabilité et donc aussi d'une moindre inaction tant des acteurs publics que des institutions religieuses, dans un effort de combinaison plus complexe d'une large liberté religieuse des organisations cultuelles avec une obligation de respecter les intérêts de leurs personnels et de contribuer à l'ordre dans la société. Une évolution qui est encore en cours...

\subsection{PERSPECTIVES D'ÉVOLUTION}

On a vu que le droit ne fournit que peu d'éléments pour définir un véritable statut satisfaisant des personnels religieux musulmans. Cette lacune aurait pu ou dû être comblée par les organisations religieuses musulmanes elles-mêmes par l'élaboration d'un «droit interne» définissant les conditions d'emploi de leurs personnels. L'État a poussé dans ce sens en incitant à la constitution de structures de gestion (CFCM, etc.). Les résultats restent cependant insatisfaisants.

Dans ces conditions, il n'est pas exclu que le législateur intervienne plus directement comme cela a été fait en Autriche ou comme cela a été proposé

31. Ainsi, dans une affaire Schüth c. Allemagne (23 sept. 2010, n 1620/03), la Cour a estimé que les conditions de licenciement d'un organiste et chef de chœur dans une paroisse catholique, révoqué pour adultère, étaient incompatibles avec la Convention.

32. Un ancien adepte mormon, engagé pour nettoyer l'église, a obtenu de la cour d'appel de Nancy l'annulation d'un licenciement motivé par son divorce (Libération, 28 juin 2001). 
par le sénateur Reichardt. La proposition de M. Reichardt tend à instituer une obligation pour les associations cultuelles de recruter leurs ministres des cultes, salariés ou bénévoles, uniquement parmi des personnes justifiant d'une qualification cultuelle reconnue. L'objectif de la mesure vise explicitement à combattre le phénomène d'imams "autoproclamés» qui développent des messages contraires à la tolérance et au respect des valeurs républicaines. L'article 2 de ce texte prévoit l'insertion suivante dans la loi du 9 décembre 1905 concernant la séparation des Églises et de l'État:

«Art. 25-2. - Les associations cultuelles ne peuvent faire appel pour l'exercice public du culte qu'à des ministres du culte justifiant d'une qualification acquise au cours d'une formation spécifique leur assurant une connaissance suffisante des principes civils et civiques ainsi que des rites de cette confession, dispensée et sanctionnée par une instance suffisamment représentative de ce culte sur le territoire national.

Pour chaque culte, les critères de cette représentativité, qui tiennent compte du nombre d'associations cultuelles qui s'en réclament, sont déterminés par décret en Conseil d'État.

Le titre de ministre du culte est ainsi conféré à toutes les personnes qui occupent, en vertu d'un contrat ou à quelque titre que ce soit et en quelque lieu que ce soit, une fonction primordiale dans la direction, le déroulement, l'animation et l'enseignement d'un culte.»

Dans ce projet, la qualification et la formation exigées sont définies par une instance cultuelle reconnue comme représentative. Cette proposition de loi n'a pas abouti, bien qu'elle ait été discutée par le Sénat ${ }^{33}$. Le texte initial a été entièrement remanié et ne subsiste plus sur le sujet du personnel religieux qu'un article relatif aux aumôniers ${ }^{34}$. Cette initiative est cependant significative

33. La Commission des lois a émis des doutes au regard de la constitutionnalité des dispositions proposées. Ces réserves constitutionnelles sont cependant restées peu circonstanciées. La Commission des lois a ainsi estimé que «les dispositions essentielles du texte soulevaient de sérieuses difficultés constitutionnelles [...] compte tenu de l'ingérence importante qu'elles impliquaient de la part de l'État dans le libre exercice des cultes et en matière de reconnaissance des cultes». On relèvera que la notion d'ingérence dans une liberté publique renvoie à la Convention européenne des droits de l'homme et non aux règles constitutionnelles. S'agissant de ces dernières, la jurisprudence du Conseil constitutionnel telle qu'elle résulte de la décision $\mathrm{n}^{\circ}$ 2010-613 DC du 7 octobre 2010 (Loi interdisant la dissimulation $d u$ visage dans l'espace public) n'exclut pas des limitations à la liberté religieuse dès lors qu'elles sont proportionnées à l'intérêt général poursuivi.

34. Art. 4 bis (nouveau): «Tout aumônier recruté par contrat, sur la proposition du culte dont il relève, atteste, dans les six mois suivant la signature de son contrat, d'une formation civile et civique, dès lors qu'il intervient: $1^{\circ}$ Dans un établissement mentionné à l'article 2 de la loi no 86-33 du 9 janvier 1986 portant dispositions statutaires relatives à la fonction publique hospitalière; $2^{\circ}$ Dans un établissement pénitentiaire mentionné à l'article 724 du code de procédure pénale; $3^{\circ}$ Dans les 
d'une préoccupation grandissante quant aux garanties que doit présenter le personnel religieux. Selon une opinion croissante, il n'y a rien de choquant en soi de procéder à une définition légale des conditions d'exercice d'une activité dès lors que ces règles respectent le libre exercice de cette activité.

Une telle intervention est cependant délicate. Aussi, la formule la plus recommandable serait de trouver des formes de réglementations consenties. D'où l'intérêt que suscite actuellement le droit local des cultes statutaires ${ }^{35}$. Ce droit se caractérise par une formule de «donnant-donnant»: une organisation religieuse peut bénéficier d'un statut particulier qui offre divers avantages (facilités juridiques, ressources financières, aide à la formation de son personnel religieux, soutien à la construction de bâtiments religieux, etc.) en échange de l'acceptation de certaines conditions de fonctionnement (notamment dans le choix et la formation des agents), de contrôles et d'une obligation de «loyauté» par rapport aux institutions publiques. Ce mécanisme ne présente pas nécessairement la forme d'une convention; il peut aussi consister en un texte législatif qui fixe le statut d'une organisation religieuse, celui-ci étant accepté par cette dernière en raison des avantages conférés.

Pour conclure, on peut estimer que la question des règles juridiques applicables aux agents des institutions religieuses est appelée à prendre une place grandissante en droit des religions. Dans ce contexte, la terminologie de «statut de ministre du culte» paraît de plus en plus anachronique et dépourvue de portée pratique. Le droit des agents des institutions religieuses en cours d'émergence est marqué par la nécessité de prendre en compte des situations diverses, des objectifs concurrents et des préoccupations pratiques variables. Il est probable, et sans doute souhaitable, que les modalités d'encadrement qui vont se développer seront diversifiées selon les organisations religieuses concernées et les responsabilités cultuelles exercées. C'est un nouveau pan du droit des religions qui se construit en la matière.

forces armées mentionnées à l'article L. 3211-1 du code de la défense.

Les aumôniers déjà en fonction lors de l'entrée en vigueur de la présente loi attestent de la formation civile et civique mentionnée au premier alinéa du présent article dans les six mois suivant la date de promulgation de la présente loi. Les modalités de la formation civile et civique mentionnée au même premier alinéa sont fixées par décret en Conseil d'État». Le texte a été transmis à l'Assemblée nationale, mais n'a pas été discuté par cette dernière: http://www.assemblee-nationale.fr/dyn/15/dossiers/justification_formation_qualifiante_exercice_culte [consulté le 15 mai 2019].

35. J.-M. Woehring, «Le culte musulman et le droit local alsacien-mosellan », Revue du droit des religions, $n^{\circ} 4,2017$, p. 141-166. 